\title{
Persistent polyclonal binucleated B-cell lymphocytosis and MECOM gene amplification
}

\author{
Edouard Cornet ${ }^{1,2^{*}}$, Hossein Mossafa ${ }^{3}$, Karine Courel $^{3}$, Jean-François Lesesve ${ }^{4}$ and Xavier Troussard ${ }^{1,2}$
}

\begin{abstract}
Background: Persistent Polyclonal Binucleated B-cell Lymphocytosis (PPBL) is characterized by a chronic polyclonal B-cell lymphocytosis with binucleated lymphocytes and a polyclonal increase in serum immunoglobulin-M. Cytogenetic is characterized by the presence of a supernumerary isochromosome $+\mathrm{i}(3)(\mathrm{q} 10)$, premature chromosome condensation and chromosomal instability. Outcome of PPBL patients is mostly benign, but subsequent malignancies could occur. The aim of our study is to provide an update of clinical and cytogenetic characteristics of our large cohort of PPBL patients, to describe subsequent malignancies occurring during the follow-up, and to investigate the role of the long arm of chromosome 3 in PPBL.

Results: We analyzed clinical, biological and cytogenetic characteristics (conventional cytogenetic analysis and fluorescent in situ hybridization) of 150 patients diagnosed with PPBL. We performed high-resolution SNP arrays in $10 \mathrm{PPBL}$ patients, comparing CD19+ versus CD19- lymphoid cells. We describe the cytogenetic characteristics in 150 PPBL patients consisting in the presence of supernumerary isochromosome +i(3)(q10) (59\%) and chromosomal instability (55\%). In CD19+ B-cells, we observed recurrent copy number aberrations of 143 genes with 129 gains (90\%) on $3 q$ and a common minimal amplified genomic region in the MECOM gene. After a median follow-up of 60 months, we observed the occurrence of 12 subsequent malignancies (12\%), 6 solid tumors and 6 Non-Hodgkin's Lymphomas, and 6 monoclonal gammopathies of undetermined significance (MGUS), requiring a long-term clinical follow-up.
\end{abstract}

Conclusions: Our clinical and cytogenetic observations lead us to hypothesize that isochromosome 3q, especially MECOM abnormality, could play a key role in PPBL.

Keywords: Persistent Polyclonal Binucleated B-cell Lymphocytosis, MECOM, SNP array

\section{Background}

Persistent Polyclonal Binucleated B-cell Lymphocytosis (PPBL) is characterized by a chronic, stable and asymptomatic lymphocytosis with binucleated lymphocytes [1]. Binucleated lymphocytes are not specific for PPBL and can be observed in patients with multiple sclerosis treated by natalizumab [2] or after accidental exposure to ionizing radiation. In the peripheral blood, a polyclonal increase of memory $\mathrm{B}$ cells $\left(\mathrm{CD} 19^{+}, \mathrm{CD} 5^{-}\right.$, $\mathrm{CD} 27^{+}, \operatorname{IgM}^{+}, \operatorname{IgD}^{+}$) is usually associated with a polyclonal increase in serum immunoglobulin-M (IgM) [36]. PPBL is characterized by a recurrent supernumerary

\footnotetext{
*Correspondence: cornet-e@chu-caen.fr

${ }^{1}$ Laboratory of Hematology, Caen University Hospital, Caen 14000, France Full list of author information is available at the end of the article
}

isochromosome $+\mathrm{i}(3)(\mathrm{q} 10)$, a premature chromosome condensation (PCC) and a chromosomal instability [3, 4]. PPBL evolution is benign in most cases, but non-Hodgkin's lymphomas and solid tumors (pulmonary blastoma) were previously and rarely described [7, 8]. In this study, we report the follow-up and the cytogenetic characteristics of a large cohort of 150 PPBL patients. We report the occurrence of subsequent malignancies in up to $12 \%$ of patients contrasting with previous studies. Strong association between supernumerary isochromosome 3q, chromosomal instability and PPBL led us to study more extensively the role of the long arm of chromosome 3 using SNP arrays in 10 patients. We observed that the $M E C O M$ gene, located on 3q26, was recurrently amplified in B-cells of PPBL patients. 


\section{Patients and methods Patients}

PPBL was diagnosed from the persistence during three months of binucleated lymphocytes on a peripheral blood film. Patients were included after written informed consent, in accordance with the Declaration of Helsinki and with institutional guidelines and after approval of the French relevant competent authorities and ethics committees (Committee of Protection of Individuals (CPP), Advisory Committee on the Processing of Information for Medical Research (CCTIRS) and the French National Commission for Data Protection (CNIL)).

Using multiparameter flow cytometry (MFC), B-cells were polyclonal in all cases, based on the expression of CD19 and the absence of a restriction of expression of light chain of immunoglobulin. Blood smears were reviewed in the same laboratory.

\section{Conventional cytogenetic analysis (CCA)}

Blood samples were collected on heparin tubes at the time of diagnosis and during the follow-up. All samples were processed in the same laboratory. CCA was performed as previously described [3]. As previously described [9], chromosomal instability was defined as the gain and/or loss of whole chromosomes or chromosomal segments at a higher rate in tumor cell population compared to normal cells.

\section{Fluorescent in situ hybridization (FISH)}

FISH was performed in order to detect supernumerary isochromosome $+\mathrm{i}(3)(\mathrm{q} 10)$ in metaphase and interphase cells using alpha-satellite chromosome 3 specific probes and Bcl6 (3q27) specific probes (Vysis ${ }^{\mathrm{TM}}$, USA). One hundred metaphases and three hundred interphases cells were analyzed per patient.

\section{SNP array}

SNP arrays were performed using Affymetrix ${ }^{\mathrm{TM}}$ Cytogenetics Whole-Genome $2.7 \mathrm{M}$ Arrays ${ }^{\circledR}$ (Affymetrix ${ }^{\mathrm{TM}}$, USA). All samples were processed in the same laboratory. Patients were selected according to the availability of sufficient fresh cells (diagnosis) or frozen cells (follow-up). Immunomagnetic sorting was performed on whole blood samples or on thawed cells in order to purify CD $19^{+}$cells (Miltenyi ${ }^{\mathrm{TM}}$ AutoMACS Pro Separator $^{\circledR}$, Bergisch Gladbach, Germany). The two fractions
$\left(\mathrm{CD} 19^{+}\right.$positive and $\mathrm{CD} 19^{-}$negative selection) were kept and the purity was checked to be $>95 \%$ by flow cytometry. The DNA was extracted from the two fractions using Gentra Puregene Blood Kit ${ }^{\circledR}$ (Qiagen ${ }^{\mathrm{TM}}$, Hilden, Germany). Hybridization of the DNA on chips was performed according the manufacturer's instructions. Chips were analyzed using Affymetrix ${ }^{\mathrm{TM}}$ Chromosome Analysis Suite ${ }^{\circledR}$ (ChASver 1.0.1). Database of annotations was NetAffx Build 30. Quality controls of the chips were set up according Affymetrix ${ }^{\mathrm{TM}}$ recommendations (SNP-QC $\geq 1.1$ and MAPD (CN$\mathrm{QC}) \leq 0.27$ ). Copy Number Aberrations (CNA) were called according user-defined thresholds (Copy Number $(\mathrm{CN})$ markers $>50$ and size $>25 \mathrm{~kb})$. The Database of Genomic Variants (DGV, http://projects.tcag.ca/ variation/) was consulted to determine whether CNA corresponded to genomic variants. Number and size of Copy Number Aberrations (CNAs) were analyzed and compared between patients and between $\mathrm{CD}_{19}{ }^{+}$ and $\mathrm{CD} 19^{-}$cells. CNA are called recurrent when at least two patients present the same CNA. Mosaicism phenomenon was detected in case of allele frequencies between disomic and trisomic states.

\section{Results}

PPBL was diagnosed in 150 untreated patients, whose main characteristics are described in Table 1. Sixtynine percent of cases showed an absolute lymphocytosis $>4 \times 10^{9} / \mathrm{L}$, with a mean percentage of binucleated lymphocytes at $3.9 \%(1-40)$. Median follow-up was 60 months (1-402) and median overall survival was not reached. Eighteen patients (12\%) developed subsequent malignancies, among which nine cases were previously described (non Hodgkin's lymphomas (NHL) in three cases, solid tumors in two cases and monoclonal gammopathies of undetermined significance (MGUS) in 4 cases) [10]. Among the 18 patients, six patients developed solid tumors with a mean time of occurrence of 87 months (3-156) (4 pulmonary cancers, 1 breast cancer and 1 cervical carcinoma). Twelve patients (8\%) developed hematological malignancies. Six cases of MGUS (IgM) (4\%) and NHL (4\%) occurred with a mean time of 75 months (0-264) and 58 months (0-120), respectively. Four patients developed a diffuse large B-cell lymphoma and 2 patients a splenic marginal zone lymphoma (Table 2 for details). Among these 18 cases, 17 patients 
Table 1 Characteristics and follow-up of 150 patients with PPBL

\begin{tabular}{|c|c|c|c|}
\hline Age (years), Mean (min-max) & $40(18.9-66.2)$ & & \\
\hline $\operatorname{Sex}(M / F)$ & $26(17 \%) / 124(83 \%)$ & & \\
\hline Tobacco consumption & 130/145 (90\%) & & \\
\hline \multicolumn{4}{|l|}{ Clinical presentation } \\
\hline Lymph node(s) & 10/108 (9 \%) & & \\
\hline Splenomegaly & 19/106 (18\%) & & \\
\hline Hepatomegaly & $2 / 108(2 \%)$ & & \\
\hline \multicolumn{4}{|l|}{ Hemogram, Mean (min-max) } \\
\hline White blood cells $\left(10^{9} / \mathrm{L}\right)$ & $12.8(7-44.8)$ & & \\
\hline Hemoglobin $(\mathrm{g} / \mathrm{dL})$ & $13.8(10.1-16.9)$ & & \\
\hline Platelets $\left(10^{9} / \mathrm{L}\right)$ & $228(83-380)$ & & \\
\hline Lymphocytosis $\left(10^{9} / \mathrm{L}\right)$ & $6.5(2.2-41)$ & & \\
\hline Binucleated Lymphocytes (\% of lymphocytes) & $3.9(1-40)$ & & \\
\hline $\operatorname{lgM}(\mathrm{g} / \mathrm{L})$, Mean (min-max) & $7.8(2.17-20)$ & & \\
\hline HLA DR7 positive & 40/52 (77\%) & & \\
\hline \multicolumn{4}{|l|}{ Multiparameter Flow Cytometry-Mean (min-max) } \\
\hline CD19 (\%) & $50.4(7-83)$ & & \\
\hline Cytogenetics & & Diagnosis & Follow-up \\
\hline +i(3)(q10) positive by karyotype & & $50 / 140(36 \%)$ & $20 / 32(63 \%)$ \\
\hline +i(3)(q10) positive by FISH & & $80 / 128(63 \%)$ & $24 / 26(92 \%)$ \\
\hline PCC positive & & $35 / 140(25 \%)$ & $8 / 32(25 \%)$ \\
\hline Chromosomal instability & & $76 / 140(54 \%)$ & $31 / 32(97 \%)$ \\
\hline Subsequent Malignancies & $18 / 150(12 \%)$ & & \\
\hline MGUS & $6 / 150(4 \%)$ & & \\
\hline Non-Hodgkin's Lymphomas & $6 / 150(4 \%)$ & & \\
\hline Solid tumors & $6 / 150(4 \%)$ & & \\
\hline
\end{tabular}

Clinical and biological data were collected from 27 centers. Median follow-up was 60 months (1-402) with unreached median overall survival MGUS monoclonal gammopathy of undetermined significance

were chronic smokers. These data strongly lead us to consider PPBL as a premalignant state requiring a longterm follow-up.

At diagnosis, CCA and FISH were performed in 140 and 128 patients, respectively. During the follow-up, CCA was performed in 32 patients $(21 \%)$. CCA and FISH detected no cytogenetic abnormality in 52/140 patients (37 \%). Recurrent supernumerary isochromosome $+\mathrm{i}(3)$ (q10) was identified in 82/140 patients (59\%). PCC, arising from asynchronous mitotic activity in multinucleated cells, was observed concomitantly with $+\mathrm{i}(3)$ (q10) in 30/140 patients (21 \%). By CCA, trisomy 8 and $\operatorname{del}(6 \mathrm{q})$ were also detected either as recurrent abnormalities (2/140 and 5/140, respectively) or as nonrecurrent abnormalities (9/140 and 4/140, respectively).
Chromosomal instability was observed in $76 / 140$ patients (54\%) and persisted in $31 / 32$ patients (97\%) during follow-up.

To determine whether $3 \mathrm{q}$ could be implicated in PPBL pathogenesis, SNP arrays were performed in 10 patients ( 3 males, 7 females) with $+\mathrm{i}(3)$ (q10) in $9 / 10$ patients (Table 3 for details). Written informed consents were obtained from the patients. The comparative analysis of sorted $\mathrm{CD} 19^{+}$and $\mathrm{CD} 19^{-}$cells revealed that CNAs were observed predominantly in $\mathrm{CD} 19^{+}$ B-cells on 3q (Table 4) with mosaicism phenomenon in 3 patients. Genetic instability was observed in all cases and predominantly in $\mathrm{CD} 19^{+}$B-cells. We observed 143 recurrent CNAs with 129 gains (90\%) on 3q of B-cells (Table 5). Interestingly, we identified with a high 
Table 2 Eighteen subsequent malignancies occurred in PPBL patients

\begin{tabular}{llll}
\hline Patients & Delay between PPBL and subsequent malignancy's diagnosis & Type of malignancy & Follow-up \\
\hline UPN36 & 38 months & DLBCL & 56 months \\
UPN47 & 20 months & SMZL & +65 months \\
UPN57 & 92 months & DLBCL & 99 months \\
UPN63 & Diagnosis of PPBL and lymphoma was concomitant & DLBCL & +13 months \\
UPN71 & 77 months & SMZL & +86 months \\
UPN83 & 120 months & DLBCL & +131 months \\
UPN1 & 264 months & MGUS & +348 months \\
UPN10 & 144 months & MGUS & +148 months \\
UPN157 & 44 months & MGUS & +47 months \\
UPN118 & Diagnosis of PPBL and MGUS was concomitant & MGUS & +36 months \\
UPN163 & Diagnosis of PPBL and MGUS was concomitant & MGUS & +57 months \\
UPN105 & Diagnosis of PPBL and MGUS was concomitant & MGUS & +42 months \\
UPN5 & 96 months & Mammary carcinoma & +272 months \\
UPN6 & 3 months & Pulmonary carcinoma & 3 months \\
UPN70 & 22 months & Pulmonary carcinoma \\
UPN86 & 132 months & Pulmonary carcinoma & +22 months \\
UPN160 & 114 months & Pulmonary carcinoma & +146 months \\
UPN67 & 156 months & Cervical carcinoma & +112 months \\
\hline
\end{tabular}

Six patients developed solid tumors ( 4 pulmonary cancers, 1 breast cancer and 1 cervical carcinoma) and 6 patients hematological malignancies (diffuse large B-cell lymphoma (DLBCL) in 4 cases, splenic marginal zone lymphoma (SMZL) in 2 cases) and 6 patients monoclonal gammopathies of undetermined significance (MGUS) $(\lg M)$

Table 3 Characteristics of the 10 patients analyzed by SNP arrays (UPN: Unique Patient Number)

\begin{tabular}{|c|c|c|c|}
\hline Patient & Karyotype & PCC (\%) & $\mathrm{FISH}+\mathrm{i}(3)(\mathrm{q} 10)(\%)$ \\
\hline UPN8 ${ }^{b}$ & $46-47, X X,+i(3)(q 10)[3] / 46, X X, \operatorname{del}(2)(q 22),-17,+\operatorname{mar}[1] / 45, X,-X[1] / 46, X X[40]$ & Absent & Present (6\%) \\
\hline UPN57 & $\begin{array}{l}47, X Y,+i(3)(q 10)[5] / 48, X Y,+i(3)(q 10),+12[01] / 46, X Y, t(14 ; 18)(q 32 ; q 22)[01] / 47, X Y, t(11 ; 14)(q 13 ; q 32) \\
\quad+\operatorname{mar}[01] / 46, X Y, \operatorname{add}(3)(p 26)[1] / 47, X Y,+22[01] / 49, X Y,+i(3)(q 10),+8,+\operatorname{mar}[01] / 46, X Y[39]\end{array}$ & Absent & Present (7\%) \\
\hline UPN136 & $46, X X[48] / P C C[2]$ & Present (4 \%) & Present (4\%) \\
\hline UPN71 ${ }^{\mathrm{C}}$ & $\begin{array}{l}\text { 47,XX, +X,del(6)(q15q26)[01]/46,XX,del(6)(q15q26),der(6)t(6;6)(q21;q23)[08]/46,XX,del(1)(q12),der(14) } \\
\quad t(1 ; 14)(p 22 ; q 32)[02] / 46, X X[09]\end{array}$ & Absent & Present (2\%) \\
\hline UPN127b & $47, X Y,+i(3)(q 10)[3] / 46, X, \operatorname{der}(Y) t(Y ; ?)(q 12 ; ?)[3] / 46, X Y[12]$ & Absent & Present (12\%) \\
\hline UPN138 & $\begin{array}{l}\text { 46,XX,del(6)(q21q24) [6] /46,XX,der(8)t(3;8)(q11;q11),der(17)t(17;?)(p11;?) [2] /46,XX,del(17)(p11) [2] } \\
\text { /46,XX,t(1;6)(q24;q21) [1]/46,XX,der(14)t(14;?)(p25;?) [1] /46,XX,dup(3)(p13p26) [1] /46,XX,der(4)t(4;?) } \\
(p 16 ; ?)[1] / 46, X X[26]\end{array}$ & Absent & Present (11\%) \\
\hline UPN99a & $47, X X,+18[2] / 47, X X,+3[1] / 46, X X[37] / P C C[1]$ & Present (2\%) & Present (4\%) \\
\hline UPN147 & $46, X X[50]$ & Absent & Absent \\
\hline UPN73a & $46, X X[50]$ & Absent & Present (1.4\%) \\
\hline UPN105a & $46, X Y[46] / 46, X Y[c p 4]$ & Absent & Present (3 \%) \\
\hline
\end{tabular}

Depending on the quality of extracted DNA, we performed DNA arrays on a both CD19+ and CD19- cells in 7 patients, ${ }^{b} \mathrm{CD} 19^{+}$cells in 2 patients and ${ }^{\mathrm{c}} \mathrm{CD} 19^{-}$cells in 1 patient. CCA and/or FISH detected $+\mathrm{i}(3)(\mathrm{q} 10)$ in $9 / 10$ patients

PCC premature chromosome condensation

frequency (7/9 patients) partial or complete amplification of one particular genomic region located in 3q26.2. The size of this common minimal amplified region was
28 kilobases (85 copy number markers) located in coding region of $M E C O M$ gene (Fig. 1). This amplification was not detected in two patients (UPN147 and UPN136). In 
Table 4 Repartition of CNAs observed in $\mathrm{CD} 19^{-}$and $\mathrm{CD} 19^{+}$cells. $\mathrm{CD} 19^{+}$cells presented twice as many $\mathrm{CNAs}$ as $\mathrm{CD} 19^{-}[83$ CNAs (12-218) versus 42 (3-184)]

\begin{tabular}{|c|c|c|c|c|c|c|c|c|c|c|}
\hline \multirow{2}{*}{$\begin{array}{l}\text { CD19- } \\
\text { Chromosome }\end{array}$} & \multicolumn{10}{|c|}{ CNAs-Total (gains/losses) } \\
\hline & UPN73 & \multicolumn{2}{|c|}{ UPN71 } & UPN57 & UPN136 & UPN138 & UPN99 & UPN147 & UPN105 & Mean \\
\hline 1 & $2(2 / 0)$ & \multicolumn{2}{|c|}{$0(0 / 0)$} & $4(1 / 3)$ & $1(0 / 1)$ & $3(3 / 0)$ & $1(0 / 1)$ & $0(0 / 0)$ & $17(0 / 17)$ & $3.5(0.7 / 2.8)$ \\
\hline 2 & $3(1 / 2)$ & \multicolumn{2}{|c|}{$1(1 / 0)$} & $0(0 / 0)$ & $2(0 / 2)$ & $2(2 / 0)$ & $0(0 / 0)$ & $0(0 / 0)$ & $24(0 / 24)$ & $4.0(0.5 / 3.5)$ \\
\hline 3 & $2(1 / 1)$ & \multicolumn{2}{|c|}{$0(0 / 0)$} & $3(3 / 0)$ & $0(0 / 0)$ & $0(0 / 0)$ & $0(0 / 0)$ & $0(0 / 0)$ & $8(1 / 7)$ & $1.6(0.6 / 1.0)$ \\
\hline $3 p$ & $0(0 / 0)$ & \multicolumn{2}{|c|}{$0(0 / 0)$} & $0(0 / 0)$ & $0(0 / 0)$ & $0(0 / 0)$ & $0(0 / 0)$ & $0(0 / 0)$ & $0(0 / 0)$ & $0.0(0.0 / 0.0)$ \\
\hline $3 q$ & $2(1 / 1)$ & \multicolumn{2}{|c|}{$0(0 / 0)$} & $3(3 / 0)$ & $0(0 / 0)$ & $0(0 / 0)$ & $0(0 / 0)$ & $0(0 / 0)$ & $8(1 / 7)$ & $1.6(0.6 / 1.0)$ \\
\hline 4 & $0(0 / 0)$ & $0(0 / 0$ & & /0) & $2(1 / 1)$ & $0(0 / 0)$ & $0(0 / 0)$ & $1(1 / 0)$ & $30(1 / 29)$ & $4.1(0.4 / 3.7)$ \\
\hline 5 & $0(0 / 0)$ & $1(0 / 1)$ & & $/ / 1)$ & $0(0 / 0)$ & $1(1 / 0)$ & $0(0 / 0)$ & $0(0 / 0)$ & $15(0 / 15)$ & $2.3(0.1 / 2.2)$ \\
\hline 6 & $0(0 / 0)$ & $0(0 / 0$ & & /0) & $0(0 / 0)$ & $1(1 / 0)$ & $0(0 / 0)$ & $0(0 / 0)$ & $11(0 / 11)$ & $1.5(0.1 / 1.4)$ \\
\hline 7 & $0(0 / 0)$ & $0(0 / 0$ & & /0) & $0(0 / 0)$ & $1(1 / 0)$ & $0(0 / 0)$ & $1(1 / 0)$ & $12(0 / 12)$ & $1.8(0.3 / 1.5)$ \\
\hline 8 & $1(0 / 1)$ & $0(0 / 0$ & & /0) & $1(1 / 0)$ & $3(3 / 0)$ & $0(0 / 0)$ & $0(0 / 0)$ & $6(0 / 6)$ & $1.4(0.5 / 0.9)$ \\
\hline 9 & $0(0 / 0)$ & $4(0 / 4)$ & & //3) & $0(0 / 0)$ & $4(2 / 2)$ & $1(1 / 0)$ & $0(0 / 0)$ & $7(0 / 7)$ & $2.4(0.4 / 2.0)$ \\
\hline 10 & $1(1 / 0)$ & $1(0 / 1)$ & & $/ / 1)$ & $0(0 / 0)$ & $1(1 / 0)$ & $0(0 / 0)$ & $1(1 / 0)$ & $7(0 / 7)$ & $1.5(0.4 / 1.1)$ \\
\hline 11 & $0(0 / 0)$ & $2(1 / 1)$ & & /0) & $0(0 / 0)$ & $2(2 / 0)$ & $0(0 / 0)$ & $1(0 / 1)$ & $10(0 / 10)$ & $1.9(0.4 / 1.5)$ \\
\hline 12 & $2(2 / 0)$ & $0(0 / 0)$ & & $/ / 1)$ & $0(0 / 0)$ & $1(1 / 0)$ & $0(0 / 0)$ & $0(0 / 0)$ & $8(1 / 7)$ & $1.5(0.5 / 1.0)$ \\
\hline 13 & $0(0 / 0)$ & $0(0 / 0)$ & & $/ 2)$ & $0(0 / 0)$ & $1(0 / 1)$ & $0(0 / 0)$ & $0(0 / 0)$ & $8(1 / 7)$ & $1.4(0.1 / 1.3)$ \\
\hline 14 & $0(0 / 0)$ & $0(0 / 0)$ & & /0) & $0(0 / 0)$ & $0(0 / 0)$ & $0(0 / 0)$ & $0(0 / 0)$ & $6(1 / 5)$ & $0.8(0.1 / 0.7)$ \\
\hline 15 & $2(2 / 0)$ & $1(1 / 0)$ & & $/ / 1)$ & $2(0 / 2)$ & $2(1 / 1)$ & $0(0 / 0)$ & $1(1 / 0)$ & $4(1 / 3)$ & $1.6(0.9 / 0.7)$ \\
\hline 16 & $1(1 / 0)$ & $0(0 / 0)$ & & & $1(1 / 0)$ & $3(1 / 2)$ & $0(0 / 0)$ & $0(0 / 0)$ & $1(0 / 1)$ & $0.8(0.4 / 0.4)$ \\
\hline 17 & $0(0 / 0)$ & $0(0 / 0)$ & & /1) & $0(0 / 0)$ & $1(1 / 0)$ & $0(0 / 0)$ & $0(0 / 0)$ & $4(2 / 2)$ & $0.8(0.4 / 0.4)$ \\
\hline 18 & $0(0 / 0)$ & $0(0 / 0)$ & & /0) & $0(0 / 0)$ & $1(1 / 0)$ & $0(0 / 0)$ & $0(0 / 0)$ & $1(0 / 1)$ & $0.2(0.2 / 0.1)$ \\
\hline 19 & $0(0 / 0)$ & $1(0 / 1)$ & & /0) & $0(0 / 0)$ & $0(0 / 0)$ & $1(0 / 1)$ & $1(0 / 1)$ & $1(0 / 1)$ & $0.5(0.0 / 0.5)$ \\
\hline 20 & $1(1 / 0)$ & $0(0 / 0)$ & & & $0(0 / 0)$ & $1(1 / 0)$ & $0(0 / 0)$ & $0(0 / 0)$ & $0(0 / 0)$ & $0.3(0.3 / 0.0)$ \\
\hline 21 & $1(0 / 1)$ & $1(0 / 1)$ & & & $0(0 / 0)$ & $0(0 / 0)$ & $0(0 / 0)$ & $0(0 / 0)$ & $1(0 / 1)$ & $0.5(0.0 / 0.5)$ \\
\hline 22 & $0(0 / 0)$ & $0(0 / 0)$ & & & $0(0 / 0)$ & $0(0 / 0)$ & $0(0 / 0)$ & $1(1 / 0)$ & $1(1 / 0)$ & $0.3(0.3 / 0.0)$ \\
\hline$x$ & $1(1 / 0)$ & $7(6 / 1)$ & & 3/0) & $1(0 / 1)$ & $25(24 / 1)$ & $0(0 / 0)$ & $2(1 / 1)$ & $1(0 / 1)$ & $6.2(5.6 / 0.6)$ \\
\hline Y & $1(1 / 0)$ & $1(1 / 0)$ & & /2) & $0(0 / 0)$ & $2(2 / 0)$ & $0(0 / 0)$ & $0(0 / 0)$ & $1(0 / 1)$ & $0.9(0.5 / 0.4)$ \\
\hline Total & $18(13 / 5)$ & $20(10 /$ & & 8/15) & $10(3 / 7)$ & $55(48 / 7)$ & $3(1 / 2)$ & $9(6 / 3)$ & $184(9 / 175)$ & $41.5(13.5 / 28)$ \\
\hline CD19+ & CNAs-tot & l (gains/l & osses) & & & & & & & \\
\hline Chromosome & UPN73 & UPN8 & UPN57 & UPN136 & UPN127 & UPN138 & UPN99 & UPN147 & UPN105 & Mean \\
\hline 1 & $20(19 / 1)$ & $0(0 / 0)$ & $0(0 / 0)$ & $4(0 / 4)$ & $0(0 / 0)$ & $0(0 / 0)$ & $5(4 / 1)$ & $8(1 / 7)$ & $0(0 / 0)$ & $5.0(3.6 / 1.4)$ \\
\hline 2 & $22(22 / 0)$ & $2(1 / 1)$ & $0(0 / 0)$ & $2(0 / 2)$ & $0(0 / 0)$ & $1(0 / 1)$ & $3(3 / 0)$ & $12(1 / 11)$ & $0(0 / 0)$ & $5.7(3.9 / 1.8)$ \\
\hline 3 & $20(19 / 1)$ & $4(4 / 0)$ & $26(25 / 1)$ & $0(0 / 0)$ & $46(46 / 0)$ & $1(1 / 0)$ & $2(2 / 0)$ & $5(0 / 5)$ & $113(113 / 0)$ & $25.0(24.2 / 0.8)$ \\
\hline $3 p$ & $11(11 / 0)$ & $0(0 / 0)$ & $1(0 / 1)$ & $0(0 / 0)$ & $0(0 / 0)$ & $0(0 / 0)$ & $0(0 / 0)$ & $0(0 / 0)$ & $0(0 / 0)$ & $1.8(1.7 / 0.1)$ \\
\hline $3 q$ & $9(8 / 1)$ & $4(4 / 0)$ & $25(25 / 0)$ & $0(0 / 0)$ & $46(46 / 0)$ & $1(1 / 0)$ & $2(2 / 0)$ & $5(0 / 5)$ & $113(113 / 0)$ & $23.2(22.6 / 0.7)$ \\
\hline 4 & $9(9 / 0)$ & $0(0 / 0)$ & $2(0 / 2)$ & $5(1 / 4)$ & $0(0 / 0)$ & $1(1 / 0)$ & $0(0 / 0)$ & $10(1 / 9)$ & $0(0 / 0)$ & $4.0(2.3 / 1.7)$ \\
\hline 5 & $14(13 / 1)$ & $0(0 / 0)$ & $0(0 / 0)$ & $2(0 / 2)$ & $0(0 / 0)$ & $0(0 / 0)$ & $0(0 / 0)$ & $5(0 / 5)$ & $1(0 / 1)$ & $3.4(2.4 / 1.0)$ \\
\hline 6 & $10(10 / 0)$ & $1(1 / 0)$ & $0(0 / 0)$ & $1(0 / 1)$ & $0(0 / 0)$ & $1(1 / 0)$ & $1(1 / 0)$ & $5(1 / 4)$ & $0(0 / 0)$ & $2.9(2.3 / 0.6)$ \\
\hline 7 & $12(12 / 0)$ & $1(0 / 1)$ & $0(0 / 0)$ & $1(0 / 1)$ & $1(0 / 1)$ & $0(0 / 0)$ & $1(1 / 0)$ & $6(1 / 5)$ & $0(0 / 0)$ & $2.4(1.6 / 0.8)$ \\
\hline 8 & $13(11 / 2)$ & $0(0 / 0)$ & $0(0 / 0)$ & $0(0 / 0)$ & $0(0 / 0)$ & $1(0 / 1)$ & $1(1 / 0)$ & $2(0 / 2)$ & $0(0 / 0)$ & $2.3(1.8 / 0.5)$ \\
\hline 9 & $14(14 / 0)$ & $0(0 / 0)$ & $0(0 / 0)$ & $4(0 / 4)$ & $0(0 / 0)$ & $5(0 / 5)$ & $1(1 / 0)$ & $4(1 / 3)$ & $0(0 / 0)$ & $3.8(2.4 / 1.4)$ \\
\hline 10 & $8(8 / 0)$ & $0(0 / 0)$ & $0(0 / 0)$ & $3(0 / 3)$ & $0(0 / 0)$ & $0(0 / 0)$ & $0(0 / 0)$ & $0(0 / 0)$ & $0(0 / 0)$ & $1.6(1.2 / 0.4)$ \\
\hline 11 & $9(9 / 0)$ & $0(0 / 0)$ & $0(0 / 0)$ & $2(0 / 2)$ & $0(0 / 0)$ & $0(0 / 0)$ & $1(1 / 0)$ & $7(0 / 7)$ & $0(0 / 0)$ & $2.8(1.8 / 1.0)$ \\
\hline 12 & $5(5 / 0)$ & $1(0 / 1)$ & $2(0 / 2)$ & $0(0 / 0)$ & $1(1 / 0)$ & $0(0 / 0)$ & $1(1 / 0)$ & $8(1 / 7)$ & $0(0 / 0)$ & $2.7(1.6 / 1.1)$ \\
\hline 13 & $4(4 / 0)$ & $0(0 / 0)$ & $1(0 / 1)$ & $2(0 / 2)$ & $0(0 / 0)$ & $1(0 / 1)$ & $0(0 / 0)$ & $2(0 / 2)$ & $1(1 / 0)$ & $1.3(0.7 / 0.6)$ \\
\hline 14 & $3(3 / 0)$ & $0(0 / 0)$ & $1(0 / 1)$ & $0(0 / 0)$ & $0(0 / 0)$ & $0(0 / 0)$ & $2(2 / 0)$ & $0(0 / 0)$ & $0(0 / 0)$ & $1.1(1.0 / 0.1)$ \\
\hline 15 & $5(5 / 0)$ & $0(0 / 0)$ & $1(1 / 0)$ & $1(0 / 1)$ & $2(0 / 2)$ & $1(0 / 1)$ & $0(0 / 0)$ & $2(1 / 1)$ & $1(1 / 0)$ & $1.8(1.2 / 0.6)$ \\
\hline
\end{tabular}


Table 4 continued

\begin{tabular}{|c|c|c|c|c|c|c|c|c|c|c|}
\hline \multirow{2}{*}{$\begin{array}{l}\text { CD19+ } \\
\text { Chromosome }\end{array}$} & \multicolumn{10}{|c|}{ CNAs-total (gains/losses) } \\
\hline & UPN73 & UPN8 & UPN57 & UPN136 & UPN127 & UPN138 & UPN99 & UPN147 & UPN105 & Mean \\
\hline$\overline{16}$ & $2(2 / 0)$ & $0(0 / 0)$ & $0(0 / 0)$ & $2(0 / 2)$ & $1(1 / 0)$ & $2(0 / 2)$ & $0(0 / 0)$ & $1(1 / 0)$ & $1(0 / 1)$ & $1.6(1.0 / 0.6)$ \\
\hline 17 & $4(4 / 0)$ & $0(0 / 0)$ & $0(0 / 0)$ & $1(0 / 1)$ & $1(1 / 0)$ & $0(0 / 0)$ & $0(0 / 0)$ & $1(1 / 0)$ & $0(0 / 0)$ & $0.9(0.8 / 0.1)$ \\
\hline 18 & $5(5 / 0)$ & $1(1 / 0)$ & $0(0 / 0)$ & $0(0 / 0)$ & $0(0 / 0)$ & $3(3 / 0)$ & $1(1 / 0)$ & $1(0 / 1)$ & $0(0 / 0)$ & $1.9(1.8 / 0.1)$ \\
\hline 19 & $1(1 / 0)$ & $0(0 / 0)$ & $0(0 / 0)$ & $0(0 / 0)$ & $1(0 / 1)$ & $0(0 / 0)$ & $0(0 / 0)$ & $1(0 / 1)$ & $0(0 / 0)$ & $0.4(0.2 / 0.2)$ \\
\hline 20 & $5(5 / 0)$ & $0(0 / 0)$ & $0(0 / 0)$ & $0(0 / 0)$ & $0(0 / 0)$ & $0(0 / 0)$ & $2(2 / 0)$ & $1(1 / 0)$ & $0(0 / 0)$ & $1.2(1.2 / 0.0)$ \\
\hline 21 & $1(0 / 1)$ & $0(0 / 0)$ & $0(0 / 0)$ & $1(0 / 1)$ & $0(0 / 0)$ & $1(0 / 1)$ & $0(0 / 0)$ & $2(0 / 2)$ & $0(0 / 0)$ & $0.6(0.0 / 0.6)$ \\
\hline 22 & $0(0 / 0)$ & $0(0 / 0)$ & $0(0 / 0)$ & $0(0 / 0)$ & $0(0 / 0)$ & $0(0 / 0)$ & $0(0 / 0)$ & $1(1 / 0)$ & $0(0 / 0)$ & $0.3(0.3 / 0.0)$ \\
\hline$x$ & $28(27 / 1)$ & $1(0 / 1)$ & $1(1 / 0)$ & $7(5 / 2)$ & $2(2 / 0)$ & $5(4 / 1)$ & $1(1 / 0)$ & $5(3 / 2)$ & $0(0 / 0)$ & $7.0(6.2 / 0.2)$ \\
\hline Y & $4(4 / 0)$ & $1(1 / 0)$ & $0(0 / 0)$ & $6(6 / 0)$ & $0(0 / 0)$ & $14(14 / 0)$ & $2(2 / 0)$ & $0(0 / 0)$ & $0(0 / 0)$ & $3.0(3.0 / 0.0)$ \\
\hline Total & $218(211 / 7)$ & $12(8 / 4)$ & $34(27 / 7)$ & $44(12 / 32)$ & $55(51 / 4)$ & $37(24 / 13)$ & $24(23 / 1)$ & $89(15 / 74)$ & $117(115 / 2)$ & $\begin{array}{l}82.7 \\
(66.6 / 16.1)\end{array}$ \\
\hline
\end{tabular}

$28 \%$ of CNAs (0-97 \%) were located on $3 q$ in $\mathrm{CD}^{+} 9^{+}$cells compared to $5 \%(0-11 \%)$ in CD19- cells (data not shown)

one of them (UPN147), no $+\mathrm{i}(3)$ (q10) was detected by CCA and/or FISH. Unfortunately, due to the mosaicism phenomenon, with less than $20 \%$ of B-cells presenting $+\mathrm{i}(3)$ (q10), gain in $M E C O M$ gene has not been confirmed yet by other molecular studies, such as quantitative PCR.

\section{Discussion}

Similar to the described link between aneuploidy, genetic instability and the development of human cancers $[11,12]$, supernumerary isochromosome $3 \mathrm{q}$ could be the cause of chromosomal instability observed in PPBL. Transfer of isochromosome $3 \mathrm{q}$ into myoblast cell line caused abnormal cytokinesis, centrosome amplification, aneuploidy and abolished G1 arrest following DNA damage. These observations might be related to an increasing expression of ATR gene located on $3 q$ [13]. Moreover, isochromosome $3 \mathrm{q}$ has been implicated in the progression of cervical carcinomas, where cells exhibiting either tetrasomy or aneusomy for chromosomes 3 and 17 increased significantly with disease progression [13-16]. Supernumerary isochromosome 3q could explain binucleated lymphocytes and chromosomal instability observed in PPBL. MECOM abnormalities, particularly the overexpression of EVI1, have been described in the pathogenesis of myeloid neoplasm such as acute myeloid leukemia and myelodysplastic syndrome, especially concerning cell-cycle disorders
[17-21]. Furthermore, as observed by Stein et al., EVI1 activation could lead to genetic instability [22]. Even if it has never been observed in lymphoid neoplasm, the potential implication of $M E C O M$ in PPBL has to be elucidated.

The link between PPBL and subsequent malignancies remains unclear and the role of tobacco is probably dominant. Majority of our patients (17/18) with subsequent malignancies were chronic smokers. We reported recently a detailed description of 2 heavy smokers patients with subsequent malignancies, UPN57 and UPN71 [23]. Tobacco use is a recognized risk factor in the development of solid tumor, such as pulmonary cancer, and also lymphoma [24]. Therefore, in PPBL, where tobacco consumption is frequent ( $90 \%$ of our cohort of 150 patients), smoking could represent a confounding factor in interpreting the link between PPBL and subsequent malignancies.

Isochromosome $3 \mathrm{q}$ has been described in cell-cycle deregulation, chromosomal instability and progression of cervical cancers. Our cytogenetic and clinical observations lead us to hypothesize that isochromosome $3 \mathrm{q}$ in B-cells plays a key role in the physiopathology and evolution of PPBL. Although isochromosome 3q has not been yet identified in tumor cells of subsequent malignancies [23], it could be implicated in chromosomal and genomic instability. This genomic instability could be part of a multistep process leading to the emergence of a malignant $B$ 
Table 5 Recurrent Copy Number Aberrations (CNA) in CD19+ B-cells. 143 CNA had been observed

\begin{tabular}{|c|c|c|c|c|c|c|c|c|}
\hline Chr & Cytoregion & Recurrence & $\begin{array}{l}\text { Recurrence } \\
\text { including } \\
\text { mosaicism }\end{array}$ & CN state & Gene & $\begin{array}{l}\text { Minimal } \\
\text { common } \\
\text { size (kbp) }\end{array}$ & $\begin{array}{l}\text { Genic region: } \\
\text { total }(\mathrm{T}) \\
\text { Exonic }(\mathrm{E}) \\
\text { Intronic }(\mathrm{I})\end{array}$ & $\begin{array}{l}\text { CNA reported } \\
\text { in DGV }\end{array}$ \\
\hline 1 & p33 & 2 & 2 & Loss & FAF1 & 31 & I & No \\
\hline 1 & p32.2 & 2 & 2 & Gain & C1orf168 & 50.2 & $\mathrm{E} / \mathrm{l}$ & No \\
\hline 2 & $\mathrm{p} 23.2$ & 2 & 2 & Gain & ALK & 62 & $E / I$ & Yes \\
\hline 2 & $\mathrm{q} 21.2-\mathrm{q} 21.3$ & 2 & 2 & Gain & MGAT5 & 72 & $E / I$ & No \\
\hline 3 & p24.2 & 2 & 2 & Gain & THRB & 55.7 & 1 & No \\
\hline 3 & $\mathrm{q} 11.2$ & 2 & 3 & Gain & LOC255025 & 50 & E/l & No \\
\hline 3 & $\mathrm{q} 12.2$ & 2 & 3 & Gain & $\mathrm{ABI3BP}$ & 143 & $E / I$ & Yes \\
\hline 3 & $\mathrm{q} 13.13$ & 2 & 3 & Gain & DZIP3 & 10.6 & 1 & No \\
\hline 3 & $\mathrm{q} 13.31$ & 2 & 3 & Gain & ZBTB20 & 39 & I & No \\
\hline 3 & q13.31 & 2 & 3 & Gain & GAP43 & 399 & T & No \\
\hline 3 & q13.31 & 2 & 3 & Gain & LSAMP & 53 & I & No \\
\hline 3 & $\mathrm{q} 13.33$ & 2 & 3 & Gain & TMEM39A & 176 & $\mathrm{~T}$ & No \\
\hline 3 & $\mathrm{q} 13.33$ & 2 & 3 & Gain & KTELC1 & 176 & T & No \\
\hline 3 & $\mathrm{q} 13.33$ & 2 & 3 & Gain & C3orf1 & 176 & $\mathrm{~T}$ & No \\
\hline 3 & $\mathrm{q} 13.33$ & 2 & 3 & Gain & CD80 & 176 & $\mathrm{~T}$ & No \\
\hline 3 & q13.33 & 2 & 3 & Gain & ADPRH & 176 & $\mathrm{~T}$ & No \\
\hline 3 & q21.1 & 2 & 3 & Gain & HSPBAP1 & 101 & $E / I$ & No \\
\hline 3 & q21.1 & 2 & 4 & Gain & DIRC2 & 101 & $T$ & No \\
\hline 3 & q21.1 & 2 & 4 & Gain & LOC100129550 & 101 & $\mathrm{~T}$ & Yes \\
\hline 3 & q21.1 & 2 & 4 & Gain & SEC22A & 114 & $\mathrm{~T}$ & No \\
\hline 3 & q21.1 & 2 & 4 & Gain & PTPLB & 125 & $\mathrm{~T}$ & No \\
\hline 3 & q21.1 & 3 & 4 & Gain & MYLK & 70 & E/I & No \\
\hline 3 & q21.1 & 2 & 4 & Gain & CCDC14 & 121 & E/I & Yes \\
\hline 3 & $\mathrm{q} 21.2$ & 2 & 4 & Gain & KALRN & 169 & $\mathrm{~T}$ & No \\
\hline 3 & $\mathrm{q} 21.2$ & 2 & 4 & Gain & UMPS & 169 & $\mathrm{~T}$ & No \\
\hline 3 & q21.2 & 2 & 4 & Gain & ZNF148 & 164 & $E / I$ & Yes \\
\hline 3 & $\mathrm{q} 21.2$ & 2 & 4 & Gain & ALDH1L1 & 171 & $E / l$ & No \\
\hline 3 & $\mathrm{q} 21.3$ & 3 & 4 & Gain & TXNRD3IT1 & 299 & $E / I$ & No \\
\hline 3 & q21.3 & 3 & 4 & Gain & CHCHD6 & 299 & $E / I$ & No \\
\hline 3 & $\mathrm{q} 21.3$ & 2 & 4 & Gain & KLHDC6 & 95 & $T$ & No \\
\hline 3 & $\mathrm{q} 21.3$ & 2 & 4 & Gain & RUVBL1 & 211 & E/l & Yes \\
\hline 3 & q21.3 & 2 & 4 & Gain & EEFSEC & 211 & $E / I$ & Yes \\
\hline 3 & $\mathrm{q} 21.3$ & 2 & 4 & Gain & GATA2 & 76 & E/I & Yes \\
\hline 3 & q21.3 & 3 & 4 & Gain & LOC90246 & 76 & $T$ & Yes \\
\hline 3 & q21.3 & 2 & 4 & Gain & C3orf27 & 120.7 & $\mathrm{~T}$ & Yes \\
\hline 3 & $\mathrm{q} 21.3$ & 2 & 4 & Gain & TMCC1 & 268 & T & No \\
\hline 3 & $\mathrm{q} 21.3$ & 2 & 4 & Gain & COL6A4P2 & 131 & $\mathrm{~T}$ & Yes \\
\hline 3 & q22.1 & 2 & 4 & Gain & MRPL3 & 62 & $E / I$ & Yes \\
\hline 3 & q22.1 & 2 & 4 & Gain & SNORA58 & 62 & $\mathrm{~T}$ & Yes \\
\hline 3 & q22.1 & 3 & 5 & Gain & CPNE4 & 46 & I & Yes \\
\hline 3 & q22.1 & 2 & 4 & Gain & CPNE4 & 155 & $E / I$ & Yes \\
\hline 3 & q22.1 & 2 & 4 & Gain & TMEM108 & 120 & 1 & Yes \\
\hline 3 & q22.1 & 2 & 4 & Gain & TOPBP1 & 69 & $E / I$ & No \\
\hline 3 & q22.1 & 2 & 4 & Gain & RYK & 225 & $\mathrm{~T}$ & No \\
\hline 3 & q22.1 & 2 & 4 & Gain & ANAPC13 & 197 & T & Yes \\
\hline 3 & q22.1 & 2 & 4 & Gain & CEP63 & 197 & $\mathrm{~T}$ & Yes \\
\hline 3 & $\mathrm{q} 22.2$ & 2 & 4 & Gain & EPHB1 & 144 & $\mathrm{E} / \mathrm{l}$ & No \\
\hline 3 & $\mathrm{q} 22.2$ & 2 & 4 & Gain & PPP2R3A & 85 & $E / I$ & No \\
\hline
\end{tabular}


Table 5 continued

\begin{tabular}{|c|c|c|c|c|c|c|c|c|}
\hline Chr & Cytoregion & Recurrence & $\begin{array}{l}\text { Recurrence } \\
\text { including } \\
\text { mosaicism }\end{array}$ & CN state & Gene & $\begin{array}{l}\text { Minimal } \\
\text { common } \\
\text { size (kbp) }\end{array}$ & $\begin{array}{l}\text { Genic region: } \\
\text { total }(\mathrm{T}) \\
\text { Exonic }(\mathrm{E}) \\
\text { Intronic }(\mathrm{I})\end{array}$ & $\begin{array}{l}\text { CNA reported } \\
\text { in DGV }\end{array}$ \\
\hline 3 & $q 22.3$ & 2 & 4 & Gain & SOX14 & 925 & $\mathrm{~T}$ & No \\
\hline 3 & $\mathrm{q} 22.3$ & 3 & 4 & Gain & CLDN18 & 121 & $\mathrm{~T}$ & Yes \\
\hline 3 & $q 22.3$ & 2 & 4 & Gain & ARMC8 & 77 & $E / l$ & Yes \\
\hline 3 & $\mathrm{q} 22.3$ & 2 & 4 & Gain & TXNDC6 & 77 & $E / I$ & Yes \\
\hline 3 & $\mathrm{q} 22.3$ & 2 & 4 & Gain & ESYT3 & 202.7 & $E / l$ & No \\
\hline 3 & $q 22.3$ & 2 & 4 & Gain & CEP70 & 202.7 & $\mathrm{~T}$ & No \\
\hline 3 & $\mathrm{q} 22.3$ & 2 & 4 & Gain & FAIM & 202.7 & $\mathrm{~T}$ & No \\
\hline 3 & $\mathrm{q} 22.3$ & 2 & 4 & Gain & PIK3CB & 202.7 & $E / I$ & No \\
\hline 3 & $q 22.3$ & 3 & 4 & Gain & LOC729627 & 193 & $\mathrm{~T}$ & No \\
\hline 3 & $q 22.3$ & 3 & 4 & Gain & LOC389151 & 193 & $\mathrm{~T}$ & No \\
\hline 3 & $q 22.3$ & 3 & 4 & Gain & FLJ46210 & 193 & $\mathrm{~T}$ & No \\
\hline 3 & $\mathrm{q} 22.3$ & 3 & 4 & Gain & BPESC1 & 193 & $\mathrm{~T}$ & No \\
\hline 3 & $\mathrm{q} 22.3$ & 2 & 4 & Gain & PISRT1 & 319 & $\mathrm{~T}$ & No \\
\hline 3 & q23 & 2 & 4 & Gain & MRPS22 & 89 & $E / I$ & No \\
\hline 3 & $q 23$ & 2 & 4 & Gain & COPB2 & 89 & $\mathrm{~T}$ & No \\
\hline 3 & $\mathrm{q} 23$ & 3 & 4 & Gain & NMNAT3 & 277.8 & $E / l$ & No \\
\hline 3 & $q 23$ & 4 & 5 & Gain & CLSTN2 & 46 & 1 & Yes \\
\hline 3 & $\mathrm{q} 23$ & 2 & 4 & Gain & TRIM42 & 443 & $\mathrm{~T}$ & Yes \\
\hline 3 & $\mathrm{q} 23$ & 2 & 4 & Gain & SLC25A36 & 443 & $\mathrm{~T}$ & Yes \\
\hline 3 & q24 & 2 & 4 & Gain & SLC9A9 & 138 & $E / I$ & Yes \\
\hline 3 & $\mathrm{q} 24$ & 2 & 4 & Gain & PLSCR4 & 47 & E/I & No \\
\hline 3 & $\mathrm{q} 24$ & 2 & 4 & Gain & PLSCR5 & 69 & $\mathrm{~T}$ & No \\
\hline 3 & $q 24$ & 2 & 4 & Gain & AGTR1 & 194 & $E / l$ & No \\
\hline 3 & q25.1 & 2 & 4 & Gain & P2RY13 & 74 & E/I & No \\
\hline 3 & $q 25.1$ & 2 & 4 & Gain & MED12L & 74 & $E / I$ & No \\
\hline 3 & q25.1 & 2 & 4 & Gain & P2RY13 & 74 & $\mathrm{~T}$ & No \\
\hline 3 & $q 25.2$ & 2 & 4 & Gain & SGEF & 364 & $E / I$ & Yes \\
\hline 3 & $q 25.2-q 25.31$ & 3 & 4 & Gain & MME & 87.4 & $E / I$ & No \\
\hline 3 & q25.32 & 2 & 4 & Gain & VEPH1 & 78 & $E / l$ & Yes \\
\hline 3 & q25.32 & 2 & 4 & Gain & C3orf55 & 78 & $E / /$ & No \\
\hline 3 & $q 25.32$ & 3 & 4 & Gain & MLF1 & 50 & $E / I$ & No \\
\hline 3 & q26.1 & 2 & 4 & Gain & C3orf57 & 120.6 & $E / I$ & No \\
\hline 3 & q26.1 & 2 & 4 & Gain & OTOL1 & 120.6 & $\mathrm{~T}$ & No \\
\hline 3 & q26.1 & 3 & 4 & Gain & $\mathrm{SI}$ & 747 & $\mathrm{~T}$ & No \\
\hline 3 & q26.1 & 3 & 4 & Gain & $\mathrm{BCHE}$ & 329 & $E / I$ & No \\
\hline 3 & q26.1 & 2 & 4 & Gain & ZBBX & 307 & $\mathrm{~T}$ & No \\
\hline 3 & $q 26.2$ & 6 & 7 & Gain & MDS1 & 28 & $E / /$ & No \\
\hline 3 & $q 26.2$ & 2 & 4 & Gain & TERC & 59 & $\mathrm{~T}$ & Yes \\
\hline 3 & $q 26.2$ & 2 & 4 & Gain & ARPM1 & 59 & $\mathrm{~T}$ & Yes \\
\hline 3 & $q 26.2$ & 2 & 4 & Gain & MYNN & 59 & $\mathrm{~T}$ & Yes \\
\hline 3 & $q 26.2$ & 2 & 4 & Gain & LRRC34 & 59 & $E / l$ & Yes \\
\hline 3 & q26.2 & 3 & 5 & Gain & TNIK & 31 & $E / l$ & No \\
\hline 3 & q26.31 & 2 & 4 & Gain & NLGN1 & 125 & 1 & Yes \\
\hline 3 & q26.31 & 2 & 4 & Gain & NLGN1 & 64 & $E / I$ & No \\
\hline 3 & q26.31 & 2 & 4 & Gain & NAALADL2 & 113 & E/I & Yes \\
\hline 3 & q26.32 & 2 & 4 & Gain & TBL1XR1 & 60 & E/I & No \\
\hline 3 & q26.32 & 2 & 4 & Gain & KCNMB2 & 121 & $E / I$ & No \\
\hline 3 & q26.33 & 2 & 4 & Gain & USP13 & 59 & $E / I$ & No \\
\hline 3 & $q 26.33$ & 2 & 4 & Gain & PEX5L & 81 & $E / I$ & No \\
\hline
\end{tabular}


Table 5 continued

\begin{tabular}{|c|c|c|c|c|c|c|c|c|}
\hline Chr & Cytoregion & Recurrence & $\begin{array}{l}\text { Recurrence } \\
\text { including } \\
\text { mosaicism }\end{array}$ & CN state & Gene & $\begin{array}{l}\text { Minimal } \\
\text { common } \\
\text { size (kbp) }\end{array}$ & $\begin{array}{l}\text { Genic region: } \\
\text { total }(\mathrm{T}) \\
\text { Exonic }(\mathrm{E}) \\
\text { Intronic }(\mathrm{I})\end{array}$ & $\begin{array}{l}\text { CNA reported } \\
\text { in DGV }\end{array}$ \\
\hline 3 & q26.33 & 2 & 4 & Gain & CCDC39 & 118 & $E / I$ & Yes \\
\hline 3 & $\mathrm{q} 27.1$ & 2 & 4 & Gain & YEATS2 & 112 & E/I & No \\
\hline 3 & q27.1 & 2 & 4 & Gain & MAP6D1 & 112 & $\mathrm{~T}$ & No \\
\hline 3 & q27.1 & 2 & 4 & Gain & PARL & 112 & $E / I$ & No \\
\hline 3 & $\mathrm{q} 27.2$ & 2 & 4 & Gain & VPS8 & 218 & $E / I$ & No \\
\hline 3 & $q 27.2$ & 2 & 4 & Gain & ETV5 & 157 & $\mathrm{~T}$ & No \\
\hline 3 & $q 27.2$ & 2 & 4 & Gain & DGKG & 157 & $E / l$ & No \\
\hline 3 & $q 27.3$ & 2 & 4 & Gain & CRYGS & 110 & $E / l$ & No \\
\hline 3 & $\mathrm{q} 27.3$ & 2 & 4 & Gain & TBCCD1 & 110 & $\mathrm{~T}$ & No \\
\hline 3 & $\mathrm{q} 27.3$ & 2 & 4 & Gain & DNAJB11 & 110 & $\mathrm{~T}$ & No \\
\hline 3 & $\mathrm{q} 27.3$ & 2 & 4 & Gain & AHSG & 110 & $\mathrm{~T}$ & Yes \\
\hline 3 & $\mathrm{q} 27.3$ & 2 & 4 & Gain & FETUB & 110 & $E / I$ & Yes \\
\hline 3 & q27.3 & 2 & 4 & Gain & ST6GAL1 & 46 & $E / I$ & Yes \\
\hline 3 & q27.3 & 2 & 4 & Gain & MASP1 & 428 & $E / I$ & No \\
\hline 3 & q27.3 & 3 & 4 & Gain & RTP4 & 148 & $\mathrm{~T}$ & No \\
\hline 3 & $\mathrm{q} 27.3$ & 2 & 4 & Gain & SST & 428 & $\mathrm{~T}$ & No \\
\hline 3 & $q 27.3$ & 2 & 4 & Gain & FLJ42393 & 191 & $\mathrm{~T}$ & Yes \\
\hline 3 & q28 & 3 & 4 & Gain & LPP & 191 & $E / l$ & Yes \\
\hline 3 & $\mathrm{q} 28$ & 2 & 4 & Gain & TP63 & 142 & $E / l$ & No \\
\hline 3 & $q 28$ & 2 & 4 & Gain & CLDN1 & 203 & $\mathrm{~T}$ & No \\
\hline 3 & $q 28$ & 2 & 4 & Gain & CLDN16 & 203 & $\mathrm{~T}$ & No \\
\hline 3 & $\mathrm{q} 28$ & 2 & 4 & Gain & TMEM207 & 203 & $\mathrm{~T}$ & No \\
\hline 3 & q29 & 2 & 4 & Gain & C3orf59 & 396 & $E / I$ & No \\
\hline 3 & $q 29$ & 2 & 4 & Gain & MGC2889 & 396 & $\mathrm{~T}$ & Yes \\
\hline 3 & $q 29$ & 2 & 4 & Gain & HRASLS & 396 & $\mathrm{~T}$ & Yes \\
\hline 3 & q29 & 2 & 4 & Gain & ATP13A5 & 396 & $E / l$ & No \\
\hline 3 & q29 & 2 & 4 & Gain & ATP13A4 & 158 & $E / I$ & Yes \\
\hline 3 & $q 29$ & 2 & 4 & Gain & OPA1 & 158 & $\mathrm{~T}$ & Yes \\
\hline 3 & $q 29$ & 2 & 4 & Gain & GP5 & 97 & $E / I$ & No \\
\hline 3 & q29 & 2 & 4 & Gain & ATP13A3 & 97 & $\mathrm{~T}$ & Yes \\
\hline 3 & $q 29$ & 2 & 4 & Gain & TM4SF19 & 87 & $E / I$ & Yes \\
\hline 3 & q29 & 2 & 4 & Gain & UBXN7 & 87 & $E / I$ & Yes \\
\hline 3 & $q 29$ & 2 & 4 & Gain & DLG1 & 240 & $E / I$ & Yes \\
\hline 3 & $q 29$ & 2 & 4 & Gain & FYTTD1 & 50 & $\mathrm{~T}$ & Yes \\
\hline 3 & $q 29$ & 2 & 4 & Gain & $\mathrm{LRCH} 3$ & 50 & $E / I$ & Yes \\
\hline 3 & $\mathrm{q} 29$ & 2 & 4 & Gain & RPL35A & 91 & E/I & Yes \\
\hline 3 & q29 & 2 & 4 & Gain & IQCG & 91 & $E / /$ & Yes \\
\hline 3 & q29 & 2 & 4 & Gain & LMLN & 91 & $\mathrm{~T}$ & Yes \\
\hline 4 & q13.3 & 2 & 2 & Gain & SLC4A4 & 46 & $E / l$ & No \\
\hline 11 & p15.1 & 2 & 2 & Gain & NELL1 & 43 & 1 & No \\
\hline 14 & q13.1 & 2 & 2 & Gain & NPAS3 & 43 & I & Yes \\
\hline 16 & p11.1 & 2 & 2 & Gain & LOC283914 & 277 & $\mathrm{~T}$ & Yes \\
\hline 21 & p11.2-p11.1 & 2 & 3 & Loss & TPTE & 107 & $\mathrm{~T}$ & Yes \\
\hline$x$ & p22.33 & 3 & 3 & Gain & DHRSX & 31 & $E / l$ & Yes \\
\hline$x$ & q12 & 2 & 2 & Gain & EDA2R & 91 & $E / l$ & Yes \\
\hline Y & q11.21 & 2 & 2 & Gain & USP9Y & 60 & $E / I$ & No \\
\hline
\end{tabular}

129 gains concerned the long arm of chromosome 3 (3q). 123 gains concerned gene coding regions. 75 CNA did not include previously reported polymorphism (Database of Genomic Variants, DGV). Gain of one exon of MDS1 (part of MECOM gene) was recurrently observed in 7 patients (including mosaicism phenomenon) Chr chromosome, Recurrence number of patients with the same CNA, CN state Copy Number state, gain or loss 


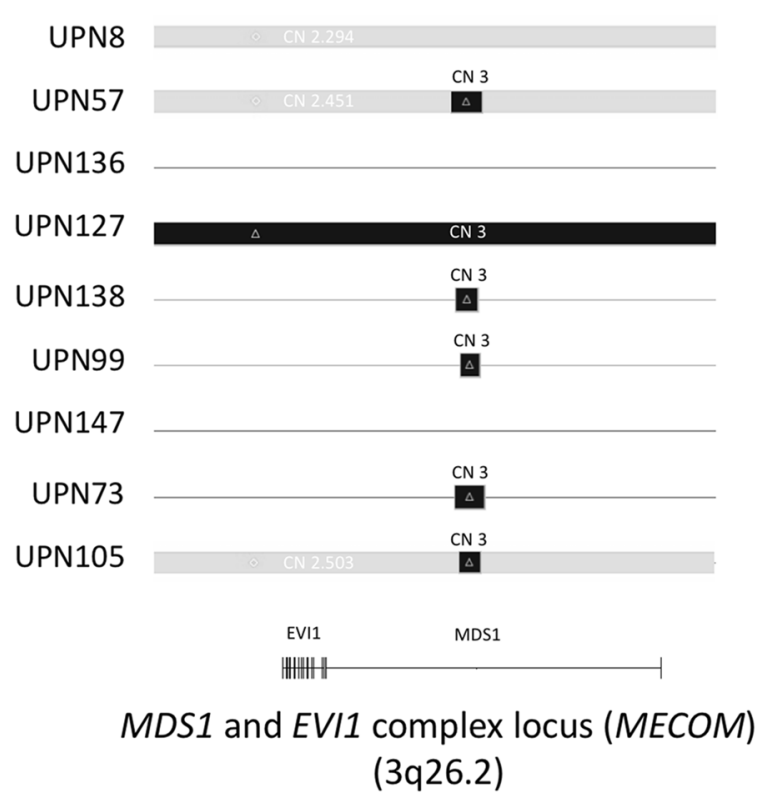

Copy Number (CN) gain:

$\triangle \mathrm{CN}=3$

$2<\mathrm{CN}<3$ (Mosaic)

Fig. 1 Schematic representation of region 3q26.2 corresponding to MECOM gene in CD19+ B-cells of 9 patients. We observed a common minimal amplified region of 28 kilobases (85 copy number markers) in 7 patients. This amplification is observed in all the CD19+ B-cells in 6 patients (copy number at $3, \mathrm{CN} 3$ ) and in a part of CD19+ B-cells in 1 patient (copy number between 2 and 3 revealing a mosaicism phenomenon)

lymphoproliferation. MECOM gene could be a good candidate to explain these observations and remains to be explored.

\section{Availability of data and materials}

All raw data are available from the authors upon request.

\section{Authors' contributions}

HM and XT designed the study. EC and XT wrote the manuscript. EC and HM analyzed SNP arrays data. KC performed SNP arrays. HM analyzed conventional cytogenetic and FISH. EC, JFL and XT examined the patients and collected clinical/biological data. All authors read and approved the final manuscript.

\section{Author details}

${ }^{1}$ Laboratory of Hematology, Caen University Hospital, Caen 14000, France. ${ }^{2}$ University of Caen, Medical School, Caen 14000, France. ${ }^{3}$ Laboratoire Cerba, Department of Genetic, Saint Ouen L'Aumone 95310, France. ${ }^{4}$ Laboratory of Hematology, Nancy University Hospital, Vandoeuvre-lès-Nancy Cedex 54511, France.

\section{Acknowledgements}

We acknowledge all the members of the Groupe Francophone d'Hématologie Cellulaire (GFHC) who participated in this study and followed up PPBL patients.

\section{Competing interests}

The authors have nothing to disclose.

Received: 2 February 2015 Accepted: 25 November 2015

Published online: 02 March 2016

\section{References}

1. Gordon DS, Jones BM, Browning SW, Spira TJ, Lawrence DN. Persistent polyclonal lymphocytosis of B lymphocytes. N Engl J Med. 1982;307(4):232-6.
2. Leclerc M, Lesesve JF, Gaillard B, Troussard X, Tourbah A, Debouverie M, Daliphard S, Delmer A. Binucleated lymphocytes in patients with multiple sclerosis treated with natalizumab. Leukemia lymphoma. 2011.

3. Mossafa H, Malaure H, Maynadie M, Valensi F, Schillinger F, Garand R, Jung G, Flandrin G, Troussard X. Persistent polyclonal B lymphocytosis with binucleated lymphocytes: a study of 25 cases. Groupe Francais d'Hematologie Cellulaire. Br J Haematol. 1999;104(3):486-93.

4. Mossafa H, Troussard X, Valensi F, Schillinger F, Maynadie M, Bulliard G, Macintyre E, Flandrin G. Isochromosome i(3q) and premature chromosome condensation are recurrent findings in chronic B-cell lymphocytosis with binucleated lymphocytes. Leukemia Lymphoma. 1996;20(3-4):267-73.

5. Himmelmann A, Gautschi O, Nawrath M, Bolliger U, Fehr J, Stahel RA. Persistent polyclonal B-cell lymphocytosis is an expansion of functional lgD(+)CD27(+) memory B cells. Br J Haematol. 2001;114(2):400-5.

6. Loembe MM, Neron S, Delage R, Darveau A. Analysis of expressed V(H) genes in persistent polyclonal $B$ cell lymphocytosis reveals absence of selection in $\mathrm{CD} 27+\operatorname{lgM}+\operatorname{lgD}+$ memory B cells. Eur J Immunol. 2002;32(12):3678-88.

7. Lawlor E, Murray M, O'Briain DS, Blaney C, Foroni L, Sarsfield P, Condell D, Sullivan F, McCann SR. Persistent polyclonal B lymphocytosis with Epstein-Barr virus antibodies and subsequent malignant pulmonary blastoma. J Clin Pathol. 1991;44(4):341-2.

8. Roy J, Ryckman C, Bernier V, Whittom R, Delage R. Large cell lymphoma complicating persistent polyclonal B cell lymphocytosis. Leukemia Off J Leukemia Soc Am Leukemia Res Fund UK. 1998;12(7):1026-30.

9. Mossafa H, Tapia S, Flandrin G, Troussard X. Chromosomal instability and ATR amplification gene in patients with persistent and polyclonal B-cell lymphocytosis (PPBL). Leukemia Lymphoma. 2004;45(7):1401-6.

10. Cornet E, Lesesve JF, Mossafa H, Sebahoun G, Levy V, Davi F, Troussard X. Long-term follow-up of 111 patients with persistent polyclonal B-cell lymphocytosis with binucleated lymphocytes. Leukemia Off J Leukemia Soc Am Leukemia Res Fund UK. 2009;23(2):419-22.

11. Duesberg P, Rausch C, Rasnick D, Hehlmann R. Genetic instability of cancer cells is proportional to their degree of aneuploidy. Proc Natl Acad Sci USA. 1998;95(23):13692-7.

12. Lengauer C, Kinzler KW, Vogelstein B. Genetic instabilities in human cancers. Nature. 1998;396(6712):643-9. 
13. Smith L, Liu SJ, Goodrich L, Jacobson D, Degnin C, Bentley N, Carr A, Flaggs G, Keegan K, Hoekstra M, et al. Duplication of ATR inhibits MyoD, induces aneuploidy and eliminates radiation-induced G1 arrest. Nat Genet. 1998;19(1):39-46.

14. Heselmeyer K, Macville M, Schrock E, Blegen H, Hellstrom AC, Shah K, Auer G, Ried T. Advanced-stage cervical carcinomas are defined by a recurrent pattern of chromosomal aberrations revealing high genetic instability and a consistent gain of chromosome arm 3q. Genes Chromosom Cancer. 1997;19(4):233-40.

15. Heselmeyer K, Schrock E, du Manoir S, Blegen H, Shah K, Steinbeck R, Auer $\mathrm{G}$, Ried T. Gain of chromosome $3 q$ defines the transition from severe dysplasia to invasive carcinoma of the uterine cervix. Proc Natl Acad Sci USA. 1996;93(1):479-84.

16. Olaharski AJ, Sotelo R, Solorza-Luna G, Gonsebatt ME, Guzman P, Mohar A, Eastmond DA. Tetraploidy and chromosomal instability are early events during cervical carcinogenesis. Carcinogenesis. 2006;27(2):337-43.

17. Buonamici S, Chakraborty S, SenyukV, Nucifora G. The role of EVI1 in normal and leukemic cells. Blood Cells Mol Dis. 2003;31(2):206-12.

18. Lugthart $S$, van Drunen $E$, van Norden $Y$, van Hoven A, Erpelinck CA, Valk PJ, Beverloo HB, Lowenberg B, Delwel R. High EVI1 levels predict adverse outcome in acute myeloid leukemia: prevalence of EVI1 overexpression and chromosome 3q26 abnormalities underestimated. Blood. 2008;111(8):4329-37.
19. Goyama S, Kurokawa M. Evi-1 as a critical regulator of leukemic cells. Int J Hematol. 2010;91(5):753-7.

20. Sitailo S, Sood R, Barton K, Nucifora G. Forced expression of the leukemiaassociated gene EVI1 in ES cells: a model for myeloid leukemia with 3q26 rearrangements. Leukemia Off J Leukemia Soc Am Leukemia Res Fund UK. 1999:13(11):1639-45.

21. Metais JY, Dunbar CE. The MDS1-EVI1 gene complex as a retrovirus integration site: impact on behavior of hematopoietic cells and implications for gene therapy. Mol Ther. 2008;16(3):439-49.

22. Stein S, Ott MG, Schultze-Strasser S, Jauch A, Burwinkel B, Kinner A, Schmidt M, Kramer A, Schwable J, Glimm H, et al. Genomic instability and myelodysplasia with monosomy 7 consequent to EVI1 activation after gene therapy for chronic granulomatous disease. Nat Med. 2010;16(2):198-204.

23. Méar J, Troussard X, Salaün V, Mossafa H, Damaj G, Le Naourès C, Galateau-Sallé F, Cornet E. Occurrence of non-hodgkin lymphoma after the diagnosis of persistent polyclonal B-cell lymphocytosis. J Leuk. 2015;3(175):2.

24. Nieters A, Deeg E, Becker N. Tobacco and alcohol consumption and risk of lymphoma: results of a population-based case-control study in Germany. Int J Cancer. 2006;1 18(2):422-30.

\section{Submit your next manuscript to BioMed Central and we will help you at every step:}

- We accept pre-submission inquiries

- Our selector tool helps you to find the most relevant journal

- We provide round the clock customer support

- Convenient online submission

- Thorough peer review

- Inclusion in PubMed and all major indexing services

- Maximum visibility for your research

Submit your manuscript at www.biomedcentral.com/submit
() Biomed Central 